\title{
X-ray Experiments for Students at the SLS Optics Beamline
}

\author{
U. Flechsig*, J. Als-Nielsen ${ }^{\dagger}$, A. Jaggi*, J. Krempaský*, P. Oberta*, S. Spielmann* \\ and J. F. van der Veen* \\ *Paul Scherrer Institut, Swiss Light Source, 5232 Villigen PSI, Switzerland \\ ${ }^{\dagger}$ University of Copenhagen, Universitetsparken 5, DK 2100 Copenhagen, Denmark
}

\begin{abstract}
We present a X-ray training course for students. The course covers fundamental properties of synchrotron radiation and basic techniques like scattering and absorption. We prepared ten experiments together with a tutorial. The whole course takes about a week. A first student group from the University of Copenhagen passed the course in June 2009. The experiments were performed at the optics beamline of the Swiss Light Source which can be part-time allocated for training purposes. Two experiments are described in more detail: scattering from a hanging drop of water turning into ice and measurement of the power of a pink synchrotron beam using a simple calorimeter.
\end{abstract}

Keywords: X-rays, scattering, diffraction, synchrotron radiation

PACS: 07.85.Qe, 42.79.Dj

\section{INTRODUCTION}

The Swiss Light Source (SLS) is an 'open access' synchrotron light source of the third generation. Beam time is granted by an international review panel and most instruments are heavily overbooked. In order to lower the barrier for students to get acquainted with the use of synchrotron radiation, SLS has organized training courses for students at various undulator beamlines during the last years. Since the start-up of the optics test beamline on a bending magnet [1], SLS offers training courses at this beamline without impacting the regular user programme.

We have designed a training course which covers fundamental properties of synchrotron radiation and basic techniques including scattering from electrons, atoms, molecules, crystals and absorption spectroscopy. We prepared ten experiments that are to easy understand. The complete course takes about a week, but students can also select fewer experiments which can be performed independent of one another. Particular emphasis is put on gaining hands-on experience with the operation of the setup and the evaluation of the data, where students are allowed to make mistakes. One of us, Jens Als-Nielsen, wrote a tutorial with the self-explanatory title "Seven Days - A Short Introduction to Modern X-Ray Science" [2] which covers the experiments and provides an introduction to the theory. The theory is treated in more detail in his book [3].

Previously the University of Copenhagen organized X-ray training courses at the second-generation synchrotron sources HASYLAB and MAXLAB. Now they follow a similar course at SLS, which runs with routine top-up injection keeping the ring current stable at $400 \pm 1 \mathrm{~mA}$. In the first half of 2009, SLS achieved an availability of $98.7 \%$ with a mean time between failures (MTBF) of 78.9 hours [4]. That is, users measure for more than 3 days without any unscheduled downtime caused by the machine.

In June 2009 a first group of sixteen students arrived. The students were split into two groups and stayed 3 days each. In order to allow the students to gain hands-on experience, we split the group further so that only two to four students stayed at the beamline at a given time while the other students prepared the experiments or performed the data evaluation outside the controls hutch of the beamline. We regard three students working simultaneously at the beamline as the optimum number. The students selected experiments from the following list:

Synchrotron radiation source. Measure the vertical profile of the bending magnet radiation. Measure the power of the bending magnet radiation in the absence of a monochromator. Compare the results with formulas given in, e.g, the "X-ray data booklet" [5].

Absorption spectroscopy. Measure the transmittance of various metal foils ( $\mathrm{Au}, \mathrm{Ag}, \mathrm{Mo}, \mathrm{Zn}, \mathrm{Cu}, \mathrm{Fe}, \mathrm{Al}$ ) as a function of photon energy. Analyze the data and show that the absorption cross section varies approximately in proportion to $1 /(\hbar \omega)^{3}$ and to $Z^{4}$.

Scattering from an electron. Measure the Compton wavelength of an electron $\lambda_{e}$ and the Thomson scattering length

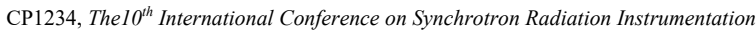
R. Garrett, I. Gentle, K. Nugent, and S. Wilkins

(C) 2010 American Institute of Physics 978-0-7354-0782-4/10/\$30.00 
(classical electron radius) $r_{e}$ by measuring the angular dependence of the scattered yield from a thin Kapton (polyimide) foil using an energy-dispersive detector. ${ }^{1}$

Scattering from an atom. Measure the angular dependence of the scattered yield from $\mathrm{Kr}$ gas in a gas cell. Determine the atomic form factor.

Scattering from a molecule. Measure the angular dependence of the scattered yield from $\mathrm{CF}_{4}$ gas in a gas cell. Determine the molecular form factor.

Reflectivity. Measure the reflectivity of an Au- coated Si wafer as a function of the grazing angle. Determine the thickness of the coating.

Resonant scattering. Measure the fluorescence from a $\beta$-brass single crystal as a function of the photon energy in the range of the absorption edges of $\mathrm{Cu}$ and $\mathrm{Zn}$ (from $8 \mathrm{keV}$ to $10.5 \mathrm{keV}$ ).

Powder diffraction. Measure the diffraction pattern from $\mathrm{Ag}$ behenate powder at various photon energies, using a hybrid pixel detector at various distances.

Liquid scattering. Measure the scattering pattern from a hanging drop of water.

Crystal diffraction. Measure the scattering pattern from the drop turned into ice.

Two experiments will be described in more detail below.

\section{THE SLS OPTICS BEAMLINE}

The SLS optics beamline has a bending magnet source and covers a photon energy range from $5.5 \mathrm{keV}$ to $22.5 \mathrm{keV}$. The beamline optics is simple: a Si (111) channel cut monochromator and a toroidal 1:1 focusing mirror providing a focus of $70 \times 140 \mu \mathrm{m} \mathrm{FWHM}(v \times h)$. Mirror and crystal can be individually retracted to allow unfocused monochromatic and pink beam operation. ${ }^{2}$ The beamline is robust and easy to operate. Basically, the student only has to switch the beam on/off and set the photon energy. For more details on the performance and instrumentation, see [1]. The experimental hutch houses an optical table, on which the hardware for the experiments is mounted. As the main hardware for the experiments we use a slit system of type 3 from JJ X-RAY, a filter wheel FRM65 from OwIS with slots for 12 metal foils for attenuation and absorption measurements and a two-circle goniometer 415 with the goniometer head 1003 from HUBER. As detectors serve a PILATUS 100k area detector, a KETEK fluorescence detector and a AXUV100 photodiode from IRD together with a KEITHLEY 6517 electrometer to measure the photocurrent. The beamline control system is implemented in EPICS. For data acquisition we use generic ioc-based scans. ${ }^{3}$

\section{DIFFRACTION FROM WATER AND ICE}

A hanging drop of water at the tip of a syringe is mounted at the diffractometer head and the diffraction pattern is recorded with the area detector. Fig. 1 shows the setup and some diffraction patterns. The students can vary the photon energy and observe the change of the ring diameter in the pattern. With a photon energy of $15 \mathrm{keV}$ and $40 \mathrm{~mm}$ distance between water drop and detector the measured diameter of the diffuse ring is $22 \mathrm{~mm}$. This corresponds to a

\footnotetext{
1 We consider the Kapton foil as a model system for an electron gas. In Thomson (elastic) scattering the scattered intensity equals
}

$$
I_{\text {scatt }}=I_{0} N r_{e}^{2} \Delta \Omega
$$

where $I_{0}$ is the incident intensity, $N$ the number of illuminated electrons and $\Delta \Omega$ the solid angle subtended by the detector. By measuring $I_{\text {scatt }}$ we can calculate $r_{e}$. The incident intensity $I_{0}$ provided by the beamline is known or can be measured with a photodiode. $N$ can be estimated from the electron density of polyimide $\left(0.47\right.$ electrons per $\left.\AA^{3}\right)$ and the illuminated volume.

The Compton wavelength of an electron $\lambda_{e}=h /\left(m_{e} c\right)$ is determined from the Compton energy shift

$$
\lambda^{\prime}-\lambda=\lambda_{e}(1-\cos \theta),
$$

where $h$ is Planck's constant, $m_{e}$ the mass of the electron, $c$ the speed of light, $\lambda^{\prime}$ and $\lambda$ the wavelength after and before scattering, respectively, and $\theta$ the scattering angle. From $r_{e}$ and $\lambda_{e}$ one can determine the fine structure constant $\alpha=r_{e}\left(\lambda_{e} / 2 \pi\right)^{-1} \approx 1 / 137$. Other fundamental constants can be deduced as well: the Bohr radius $a_{0}=r_{e} \alpha^{-2}$ and the Rydberg constant $R_{\infty}=\alpha^{2}\left(2 \lambda_{e}\right)^{-1}$.

2 We call it pink beam since the "white" bending magnet spectrum is filtered by two diamond windows and one Kapton window.

3 The scans automatically save the data at the beamline file server under the user account of the students. The students can mount this account on their laptop and analyze the data using their own software. 

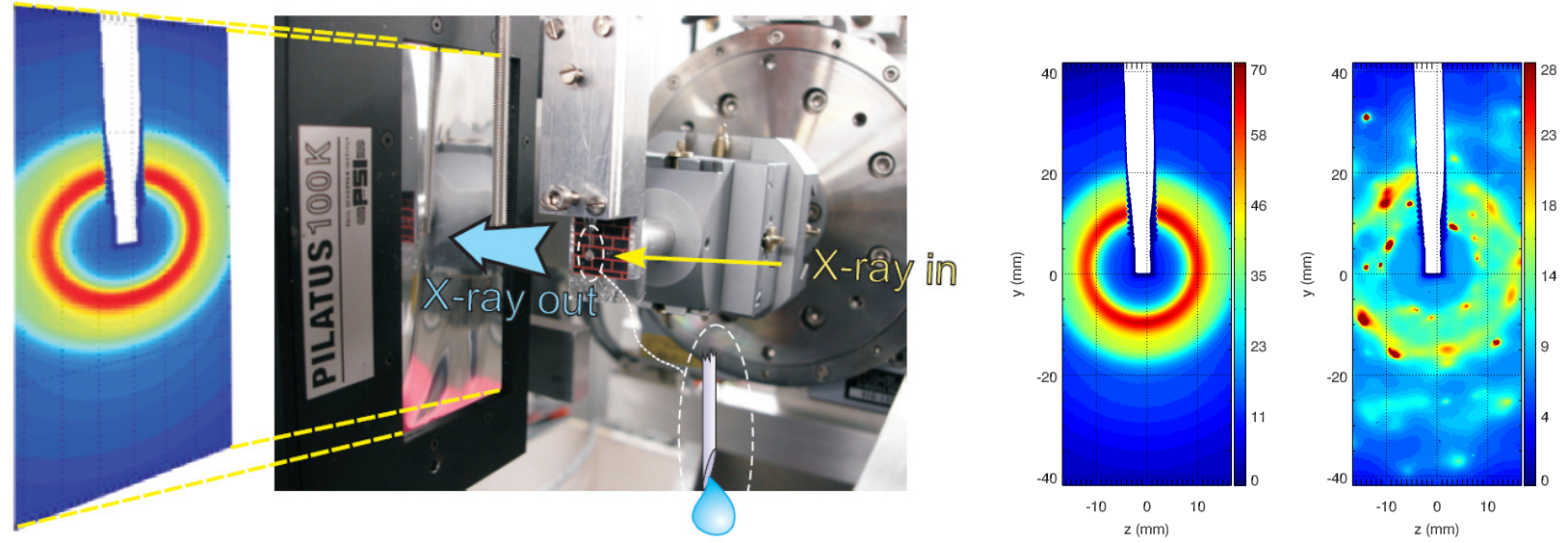

FIGURE 1. Diffraction from a hanging drop of water and ice. Left: photograph of the setup with the water drop in front of the Pilatus 100k area detector. Center: diffraction pattern from water. Right: diffraction pattern from ice. Photon energy $15 \mathrm{keV}, 40 \mathrm{~mm}$ distance between water/ice drop and detector, $1 \mathrm{~s}$ acquisition time. Color bar unit: kcounts/pixel. Pixel size: $172 \mu \mathrm{m} \times 172 \mu \mathrm{m}$.

characteristic length of $0.3 \mathrm{~nm}$. The students can be asked to perform a plausibility check using the density and molar mass of water. ${ }^{4}$

Next we freeze the water drop with a cryojet. The rings split up in separate spots which indicate the formation of crystalline ice. Indexing of the pattern is not part of the exercise but should motivate the students to go deeper into the field of crystallography. In an upgraded version of the experiment we plan to trigger the melting and freezing by remote control. ${ }^{5}$ Then we can use the multiple-image feature of PILATUS to make a movie of the diffraction patterns while the water drop is freezing or melting.

\section{POWER MEASUREMENT WITH CALORIMETER}

We measure the power of the synchrotron radiation generated by the bending magnet using the simple calorimeter shown in Fig. 2. The calorimeter consists of a small copper block $(10 \mathrm{~mm} \times 10 \mathrm{~mm} \times 5 \mathrm{~mm})$ which is held in place by thin stainless steel wires within an aluminum frame. The temperature of the copper block is measured with a thermocouple of type $\mathrm{K}$. The mass of the copper block has been determined with an analytical balance with high precision. The $5 \mathrm{~mm} \mathrm{Cu}$ thickness ensures that all the power from the bending magnet is absorbed. ${ }^{6}$

We measure the temperature while switching the beam on and off. The measurement is shown in the right panel of Fig. 2. The temperature rises linearly after the beam is switched on. After switching the beam off the temperature decreases due to the thermal losses. The temperature change $d T / d t$ is proportional to the power $d Q / d t$ :

$$
\frac{d Q}{d t}=m c \frac{d T}{d t}
$$

where $m$ is the mass and $c$ the specific heat of the copper block. $m c$ is the calorimeter constant which in our case equals $1.53 \mathrm{~J} / \mathrm{K}$. If we take the losses into account, it follows that

$$
\frac{d Q}{d t}=m c\left(\frac{d T_{1}}{d t}-\frac{d T_{2}}{d t}\right)
$$

\footnotetext{
${ }^{4}$ The density of water is $1 \mathrm{~g} / \mathrm{cm}^{3}$. The molar mass of $\mathrm{H}_{2} \mathrm{O}$ is $18 \mathrm{~g} / \mathrm{mol}$. The mass of one molecule is therefore $18 / 6 \times 10^{23}=3 \times 10^{-23} \mathrm{~g}$. From the density we conclude that one molecule occupies a volume of $3 \times 10^{-23} \mathrm{~cm}^{3}$ or $30 \times 10^{-30} \mathrm{~m}^{3}$. Assuming that water consists of point masses in a cubic grid with the lattice constant $d$, then we find $d=\sqrt[3]{30 \times 10^{-30}}=3.1 \times 10^{-10} \mathrm{~m}$, which is in good agreement with our measurement.

${ }^{5}$ We did promising tests with a commercially available Peltier element.

${ }^{6}$ The transmittance of copper at $50 \mathrm{keV}$ equals $3 \times 10^{-5}$, i.e., the transmittance for photons with $E<50 \mathrm{keV}$ is negligible.
} 

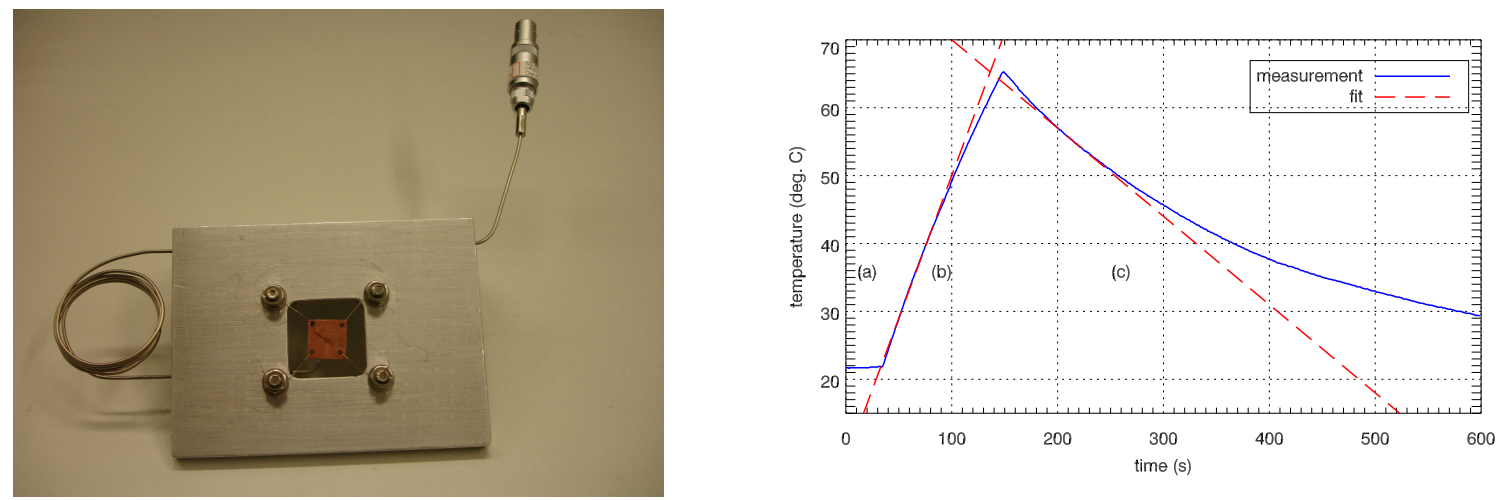

FIGURE 2. Measurement of the power of the pink beam using a simple calorimeter. Left: the calorimeter. Right: temperature of the calorimeter as function of time. We can distinguish three phases: (a) beam off, constant temperature and calorimeter in thermal equilibrium with the environment, (b) beam on, temperature rise due to absorbed power, (c) beam off, temperature decrease due to thermal losses (heat conduction, convection).

Here, $d T_{1} / d t$ and $d T_{2} / d t$ are the upward and downward temperature slopes as indicated in Fig. 2. From the plot we determine the slopes to be $0.36 \mathrm{~K} / \mathrm{s}$ and $-0.16 \mathrm{~K} / \mathrm{s}$. The result is a power of $0.8 \mathrm{~W}$. This agrees surprisingly well with the theoretical prediction of $0.72 \mathrm{~W}$, taking into account the known horizontal opening angle of $1 \mathrm{mrad}$ and the transmittance of the diamond windows, etc. The students may be asked to predict the power of the pink beam based on the formulas from the X-ray data booklet [5].

\section{CONCLUSIONS}

At the SLS optics beamline we offer X-ray training courses for students, independent of general user operation of the facility. About ten basic experiments have been prepared and a tutorial is available.

\section{REFERENCES}

1. U. Flechsig, A. Jaggi, S. Spielmann, H. A. Padmore, and A. A. MacDowell, Nuclear Inst. and Methods in Physics Research, A (2009).

2. J. Als-Nielsen, Seven Days - A Short Introduction to Modern X-Ray Science (2009), URL http://sls.web.psi.ch/ view.php/beamlines/optics/SevenDays.pdf $\%$.

3. J. Als-Nielsen, and D. McMorrow, Elements of Modern X-Ray Physics, Wiley, 2001.

4. A. Lüdecke, personal communication (2009).

5. A. Thompson, et al., X-ray data booklet (2001), URL http: / / xdb. lbl.gov. 